\title{
ON BI-HARMONIC HYPERSURFACES IN EUCLIDEAN SPACE OF ARBITRARY DIMENSION
}

\author{
RAM SHANKAR GUPTA \\ University School of Basic and Applied Sciences, \\ Guru Gobind Singh Indraprastha University, \\ Sector-16C, Dwarka, New Delhi-110078, India \\ E-mail: ramshankar.gupta@gmail.com
}

(Received 22 October 2013; revised 9 January 2014; accepted 1 April 2014; first published online 18 December 2014)

\begin{abstract}
The following Chen's bi-harmonic conjecture made in 1991 is wellknown and stays open: The only bi-harmonic submanifolds of Euclidean spaces are the minimal ones. In this paper, we prove that the bi-harmonic conjecture is true for biharmonic hypersurfaces with three distinct principal curvatures of a Euclidean space of arbitrary dimension.
\end{abstract}

2000 Mathematics Subject Classification. 53D12, 53C40, 53C42

1. Introduction. The study of submanifolds with harmonic mean curvature vector field was initiated by B.Y. Chen in 1985 and arose in the context of his theory of submanifolds of finite type. For a survey on submanifolds of finite type and various related topics was presented in (cf. [3, 4]). Let $M^{n}$ be an $n$-dimensional, connected submanifold of the Euclidean space $E^{m}$. Denote by $\vec{x}, \vec{H}$, and $\triangle$ respectively the position vector field of $M^{n}$, the mean curvature vector field of $M^{n}$, and the Laplace operator on $M^{n}$, with respect to the Riemannian metric $g$ on $M^{n}$, induced from the Euclidean metric of the ambient space $E^{m}$. Then, as it is well known, (cf. [1])

$$
\triangle \vec{x}=-n \vec{H}
$$

This shows, in particular, that $M^{n}$ is a minimal submanifold of $E^{m}$ if and only if its coordinate functions are harmonic (i.e., they are eigenfunctions of $\Delta$ with eigenvalue $0)$. We also see that every minimal submanifold of $E^{m}$ satisfies

$$
\triangle \vec{H}=0 .
$$

A submanifold $M^{n}$ of $E^{m}$ satisfying this condition (1.2) is said to have harmonic mean curvature vector field. In view of (1.1), submanifolds with harmonic mean curvature vector field are equivalently characterised by the condition

$$
\triangle^{2} \vec{x}=0
$$

Therefore, submanifolds satisfying (1.2) are also called bi-harmonic submanifolds. As remarked, minimal submanifolds are immediately seen to be bi-harmonic. Conversely, the question arises whether the class of submanifolds with harmonic mean curvature vector field is essentially larger than the class of minimal submanifolds. Otherwise stated, we consider the problem to determine, if there exist bi-harmonic 
submanifolds of $E^{m}$, other than the minimal ones. Concerning this problem B. Y. Chen conjectured the following:

Conjecture The only bi-harmonic submanifolds of Euclidean spaces are the minimal ones.

In Euclidean spaces, we have the following results, which indeed support the above mentioned conjecture. B. Y. Chen proved in 1985 that every bi-harmonic surface in $E^{3}$ is minimal. Thereafter, I. Dimitric generalised this result [7, 8] and proved that a bi-harmonic submanifold $M^{n}$ of a Euclidean space $E^{m}$ is minimal if it is one of the following:

(a) a curve,

(b) a submanifold with constant mean curvature,

(c) a hypersurface with at most two distinct principal curvatures,

(d) a pseudo-umbilical submanifold of dimension $n=4$,

(e) a submanifold of finite type.

In [9] it was proved that every bi-harmonic hypersurface in $E^{4}$ is minimal. Further, Chen and Munteanu [5] proved that the bi-harmonic conjecture is true for $\delta(2)$-ideal and $\delta(3)$-ideal hypersurfaces of a Euclidean space of arbitrary dimension. Recently, it was proved that every bi-harmonic hypersurfaces with three distinct principal curvaturesn in $E^{5}$ is minimal [10]. In this paper we have proved that Chen's conjecture is true for bi-harmonic hypersurfaces with three distinct principal curvatures in Euclidean spaces $E^{n+1}$ of arbitrary dimension.

2. Preliminaries. Let $(M, g)$ be a $n$-dimensional hypersurface isometrically immersed in a $(n+1)$-dimensional Euclidean space $\left(E^{n+1}, \bar{g}\right)$ and $g=\bar{g}_{\mid M}$.

Let $\bar{\nabla}$ and $\nabla$ denote linear connections on $E^{n+1}$ and $M$, respectively. Then, the Gauss and Weingarten formulae are given by

$$
\begin{aligned}
& \bar{\nabla}_{X} Y=\nabla_{X} Y+h(X, Y), \quad \forall X, Y \in \Gamma(T M), \\
& \bar{\nabla}_{X} \xi=-A_{\xi} X, \quad \forall \xi \in \Gamma\left(T M^{\perp}\right),
\end{aligned}
$$

where $h$ is the second fundamental form and $A$ is the shape operator. It is well known that the second fundamental form $h$ and shape operator $A$ are related by

$$
\bar{g}(h(X, Y), \xi)=g\left(A_{\xi} X, Y\right) .
$$

The mean curvature vector is given by

$$
\vec{H}=\frac{1}{n} \text { trace } h
$$

The Gauss and Codazzi equations are given by

$$
\begin{aligned}
R(X, Y) Z & =g(A Y, Z) A X-g(A X, Z) A Y, \\
\left(\nabla_{X} A\right) Y & =\left(\nabla_{Y} A\right) X,
\end{aligned}
$$


respectively, where $R$ is the curvature tensor, $A=A_{\xi}$ for some unit normal vector field $\xi$ and

$$
\left(\nabla_{X} A\right) Y=\nabla_{X}(A Y)-A\left(\nabla_{X} Y\right)
$$

for all $X, Y, Z \in \Gamma(T M)$.

A bi-harmonic submanifold in a Euclidean space is called proper bi-harmonic if it is not minimal. The necessary and sufficient conditions for $M^{n}$ to be bi-harmonic in $E^{n+1}[1]$ is

$$
\begin{aligned}
\Delta H+H \operatorname{trace} A^{2} & =0, \\
2 A(\operatorname{grad} H)+n H \operatorname{grad} H & =0,
\end{aligned}
$$

where $H$ denotes the mean curvature. Also the Laplace operator $\Delta$ of a scalar valued function $f$ is given by [1]

$$
\triangle f=-\sum_{i=1}^{n}\left(e_{i} e_{i} f-\nabla_{e_{i}} e_{i} f\right)
$$

where $\left\{e_{1}, e_{2}, \ldots, e_{n}\right\}$ is an orthonormal local tangent frame on $M^{n}$.

3. Bi-harmonic hypersurfaces with three distinct principal curvatures. In this section we study bi-harmonic hypersurfaces $M^{n}$ of $E^{n+1}$. We also assume that mean curvature is not constant. From (2.9), it is easy to see that $\operatorname{grad} H$ is an eigenvector of the shape operator $A$ with the corresponding principal curvature $\frac{-n H}{2}$. We choose $e_{1}$ in the direction of grad $H$ and therefore shape operator $A$ of hypersurfaces will take the following form with respect to a suitable frame $\left\{e_{1}, e_{2}, \ldots, e_{n-1}, e_{n}\right\}$

$$
A_{H}=\left(\begin{array}{cccccc}
\frac{-n H}{2} & & & & & \\
& \lambda_{2} & & & & \\
& & \ldots & & & \\
& & & \ldots & & \\
& & & & \lambda_{n-1} & \\
& & & & & \lambda_{n}
\end{array}\right) .
$$

The $\operatorname{grad} H$ can be expressed as

$$
\operatorname{grad} H=\sum_{i=1}^{n} e_{i}(H) e_{i}
$$

As we have taken $e_{1}$ parallel to $\operatorname{grad} H$, consequently

$$
e_{1}(H) \neq 0, e_{2}(H)=0, e_{3}(H)=0, \ldots, e_{n-1}(H)=0, e_{n}(H)=0 .
$$

We express

$$
\nabla_{e_{i}} e_{j}=\sum_{k=1}^{n} \omega_{i j}^{k} e_{k}, \quad \quad i, j=1,2, \ldots, n
$$


Using (3.4) and the compatibility conditions $\left(\nabla_{e_{k}} g\right)\left(e_{i}, e_{i}\right)=0$ and $\left(\nabla_{e_{k}} g\right)\left(e_{i}, e_{j}\right)=0$, we obtain

$$
\omega_{k i}^{i}=0, \quad \omega_{k i}^{j}+\omega_{k j}^{i}=0,
$$

for $i \neq j$, and $i, j, k=1,2, \ldots, n$.

Taking $X=e_{i}, Y=e_{j}$ in (2.7) and using (3.1), (3.4), we get

$$
\left(\nabla_{e_{i}} A\right) e_{j}=e_{i}\left(\lambda_{j}\right) e_{j}+\sum_{k=1}^{n} \omega_{i j}^{k} e_{k}\left(\lambda_{j}-\lambda_{k}\right)
$$

Putting the value of $\left(\nabla_{e_{i}} A\right) e_{j}$ in (2.6), we find

$$
e_{i}\left(\lambda_{j}\right) e_{j}+\sum_{k=1}^{n} \omega_{i j}^{k} e_{k}\left(\lambda_{j}-\lambda_{k}\right)=e_{j}\left(\lambda_{i}\right) e_{i}+\sum_{k=1}^{n} \omega_{j i}^{k} e_{k}\left(\lambda_{i}-\lambda_{k}\right),
$$

whereby for $i \neq j=k$ and $i \neq j \neq k$, we obtain

$$
\begin{aligned}
e_{i}\left(\lambda_{j}\right) & =\left(\lambda_{i}-\lambda_{j}\right) \omega_{j i}^{j}, \\
\left(\lambda_{i}-\lambda_{j}\right) \omega_{k i}^{j} & =\left(\lambda_{k}-\lambda_{j}\right) \omega_{i k}^{j},
\end{aligned}
$$

respectively, for distinct $i, j, k=1,2, \ldots, n$.

Since $\lambda_{1}=\frac{-n H}{2}$, from (3.3), we get

$$
e_{1}\left(\lambda_{1}\right) \neq 0, e_{2}\left(\lambda_{1}\right)=0, e_{3}\left(\lambda_{1}\right)=0, \ldots, e_{n-1}\left(\lambda_{1}\right)=0, e_{n}\left(\lambda_{1}\right)=0 .
$$

Using (3.8), we have

$$
\left[e_{i}, e_{j}\right]\left(\lambda_{1}\right)=0, \quad i, j=2, \ldots, n,
$$

whereby using (3.4), we find

$$
\omega_{i j}^{1}=\omega_{j i}^{1}
$$

for $i \neq j$ and $i, j=2, \ldots, n$.

Now we show that $\lambda_{j} \neq \lambda_{1}, j=2,3, \ldots, n$. In fact, if $\lambda_{j}=\lambda_{1}$ for $j \neq 1$, from (3.6), we find

$$
e_{1}\left(\lambda_{j}\right)=\left(\lambda_{1}-\lambda_{j}\right) \omega_{j 1}^{j}=0
$$

which contradicts the first expression of (3.8).

Since $M^{n}$ has three distinct principal curvatures, we can assume that $\lambda_{2}=\lambda_{3}=$ $\ldots=\lambda_{n-1}=\lambda \neq \lambda_{n}$. From (2.4), we obtain that

$$
\lambda_{n}=\frac{3 n H}{2}-(n-2) \lambda, \quad \lambda \neq \frac{-n H}{2}, \frac{2 n H}{n-2}, \frac{3 n H}{2(n-1)} .
$$

Putting $i, j=2,3, \ldots, n-1$, and $i \neq j$ in (3.6), we get

$$
e_{j}(\lambda)=0, \quad \text { for } \quad j=2,3, \ldots, n-1 .
$$

Putting $i \neq 1, j=1$ in (3.6) and using (3.8) and (3.5), we find

$$
\omega_{1 i}^{1}=0, \quad i=1,2,3, \ldots, n .
$$


Putting $i=2,3, \ldots, n-1, j=n$ in (3.6) and using (3.12), we obtain

$$
\omega_{n i}^{n}=0, \quad i=2,3, \ldots, n-1 .
$$

Putting $i=1, j=2,3, \ldots, n-1, n$, in (3.6), we have

$$
\omega_{n 1}^{n}=\frac{e_{1}(3 n H-2(n-2) \lambda)}{-4 n H+2(n-2) \lambda}, \quad \omega_{j 1}^{j}=-\frac{2 e_{1}(\lambda)}{n H+2 \lambda}, \quad j=2,3, \ldots, n-1 .
$$

Putting $i=n, j=2,3, \ldots, n-1$, in (3.6), we find

$$
\omega_{j n}^{j}=\frac{2 e_{n}(\lambda)}{3 n H-2(n-1) \lambda}, \quad j=2,3, \ldots, n-1 .
$$

Putting $i=1, j \neq k$, and $j, k=2,3, \ldots, n-1$, in (3.7), we obtain

$$
\omega_{k 1}^{j}=0, \quad j \neq k, \quad \text { and } \quad j, k=2,3, \ldots, n-1 .
$$

Putting $i=n, j \neq k$, and $j, k=2,3, \ldots, n-1$, in (3.7), we have

$$
\omega_{k n}^{j}=0, \quad j \neq k, \quad \text { and } \quad j, k=2,3, \ldots, n-1 .
$$

Putting $i=n, j=1$, and $k=2,3, \ldots, n-1$, in (3.7), and using (3.9) we get

$$
\omega_{k n}^{1}=\omega_{n k}^{1}=0, \quad k=2,3, \ldots, n-1 .
$$

Putting $i=1, j=n$, and $k=2,3, \ldots, n-1$, in (3.7), and using (3.9) we find

$$
\omega_{1 k}^{n}=\omega_{k 1}^{n}=0, \quad k=2,3, \ldots, n-1 .
$$

Now, we have the following:

LEMMA 3.1. Let $M^{n}$ be an n-dimensional bi-harmonic hypersurface with non-constant mean curvature in Euclidean space $E^{n+1}$, having the shape operator given by (3.1) with respect to suitable orthonormal frame $\left\{e_{1}, e_{2}, \ldots, e_{n-1}, e_{n}\right\}$. Then, we obtain

$$
\begin{aligned}
& \nabla_{e_{1}} e_{1}=0, \quad \nabla_{e_{i}} e_{1}=-\alpha e_{i}, i=2,3, \ldots, n-1, \quad \nabla_{e_{n}} e_{1}=\beta e_{n}, \\
& \nabla_{e_{i}} e_{i}=\alpha e_{1}+\sum_{i \neq j, j=2}^{n-1} \omega_{i i}^{j} e_{j}-\frac{2 e_{n}(\lambda)}{3 n H-2(n-1) \lambda} e_{n}, \quad i=2,3, \ldots, n-1, \\
& \nabla_{e_{i}} e_{j}=\sum_{i \neq j, k=2}^{n-2} \omega_{i j}^{k} e_{k}, \quad i, j=2,3, \ldots, n-1, \\
& \nabla_{e_{1}} e_{n}=0, \quad \nabla_{e_{n}} e_{n}=-\beta e_{1}, \quad \nabla_{e_{i}} e_{n}=\frac{2 e_{n}(\lambda)}{3 n H-2(n-1) \lambda} e_{i}, \quad i=2,3, \ldots, n-1,
\end{aligned}
$$

where $\omega_{i j}^{k}$ satisfies (3.5) for $i, j, k=1,2,3, \ldots, n-1, n$, and $\alpha=\frac{2 e_{1}(\lambda)}{n H+2 \lambda}, \beta=$ $\frac{e_{1}(3 n H-2(n-2) \lambda)}{-4 n H+2(n-2) \lambda}$.

Using Lemma 3.1, Gauss equation and comparing the coefficients with respect to a orthonormal frame $\left\{e_{1}, e_{2}, \ldots, e_{n-1}, e_{n}\right\}$, we find the following: 
- $X=e_{1}, Y=e_{2}, Z=e_{1}$,

$$
-e_{1}(\alpha)+\alpha^{2}=\frac{n H \lambda}{2} .
$$

- $X=e_{1}, Y=e_{2}, Z=e_{n}$,

$$
e_{1}\left(\frac{2 e_{n}(\lambda)}{3 n H-2(n-1) \lambda}\right)-\alpha \frac{2 e_{n}(\lambda)}{3 n H-2(n-1) \lambda}=0 .
$$

- $X=e_{1}, Y=e_{n}, Z=e_{1}$,

$$
e_{1}(\beta)+\beta^{2}=\frac{n H}{2}\left(\frac{3 n H}{2}-(n-2) \lambda\right) .
$$

- $X=e_{3}, Y=e_{n}, Z=e_{1}$,

$$
e_{n}(\alpha)+\frac{2 e_{n}(\lambda)}{3 n H-2(n-1) \lambda}(\alpha+\beta)=0 .
$$

- $X=e_{n}, Y=e_{2}, Z=e_{n}$,

$$
e_{n}\left(\frac{2 e_{n}(\lambda)}{3 n H-2(n-1) \lambda}\right)-\alpha \beta-\left(\frac{2 e_{n}(\lambda)}{3 n H-2(n-1) \lambda}\right)^{2}=-\lambda\left(\frac{3 n H}{2}-(n-2) \lambda\right) .
$$

Using (2.8), (2.10), (3.1) and Lemma 3.1, we find

$$
-e_{1} e_{1}(H)+[(n-2) \alpha-\beta] e_{1}(H)+H\left[\frac{n^{2} H^{2}}{4}+(n-2) \lambda^{2}+\left(\frac{3 n H}{2}-(n-2) \lambda\right)^{2}\right]=0 .
$$

From (3.3) and Lemma 3.1, we obtain

$$
e_{i} e_{1}(H)=0, \quad i=2,3, \ldots, n-1, n .
$$

Differentiating $\alpha=\frac{2 e_{1}(\lambda)}{n H+2 \lambda}, \beta=\frac{e_{1}(3 n H-2(n-2) \lambda)}{-4 n H+2(n-2) \lambda}$ along $e_{n}$, we get equations

$$
\begin{gathered}
(n H+2 \lambda) e_{n}(\alpha)+2 \alpha e_{n}(\lambda)=2 e_{n} e_{1}(\lambda), \\
(-4 n H+2(n-2) \lambda) e_{n}(\beta)=-2(n-2) e_{n} e_{1}(\lambda)-2(n-2) \beta e_{n}(\lambda)
\end{gathered}
$$

respectively and eliminating $e_{n} e_{1}(\lambda)$, we have

$$
(-4 n H+2(n-2) \lambda) e_{n}(\beta)=-(n-2)(n H+2 \lambda) e_{n}(\alpha)-2(n-2)(\alpha+\beta) e_{n}(\lambda) .
$$

Putting the value of $e_{n}(\alpha)$ from (3.28) in the above equation, we find

$$
e_{n}(\beta)=\frac{4 e_{n}(\lambda) n(n-2)(\alpha+\beta)(\lambda-H)}{(-4 n H+2(n-2) \lambda)(3 n H-(2 n-2) \lambda)}
$$

Differentiating (3.30) along $e_{n}$ and using (3.31), (3.28) and $e_{n}(\beta)$, we get

$$
e_{n}(\lambda)\left[\frac{4(\alpha+\beta) e_{1}(H)}{-4 n H+2(n-2) \lambda}+H((2 n-2) \lambda-3 n H)\right]=0 \text {. }
$$


We claim that $e_{n}(\lambda)=0$. Indeed, if $e_{n}(\lambda) \neq 0$, then

$$
\frac{4(\alpha+\beta) e_{1}(H)}{-4 n H+2(n-2) \lambda}+H((2 n-2) \lambda-3 n H)=0
$$

Now, differentiating (3.33) along $e_{n}$, we have

$$
\frac{8(\alpha+\beta)(n H(14-5 n)+4(n-2)(n-1) \lambda) e_{1}(H)}{(-4 n H+2(n-2) \lambda)^{2}\left(\frac{3 n H}{2}-(n-2) \lambda\right)}+H((2 n-2)=0 .
$$

Eliminating $e_{1}(H)$ from (3.33) and (3.34), we obtain

$$
2(n-1) \lambda-3 n H=0
$$

which is not possible since $\lambda \neq \frac{3 n H}{2(n-1)}$, consequently, $e_{n}(\lambda)=0$. Therefore, (3.29) reduces to

$$
\alpha \beta=\lambda\left(\frac{3 n H}{2}-(n-2) \lambda\right)
$$

Now, eliminating $e_{1} e_{1}(H)$ and $e_{1} e_{1}(\lambda)$, using (3.35), (3.30), (3.27) and (3.25), we obtain

$$
\left[\left(10 n-2 n^{2}\right) \alpha-4 n \beta\right] e_{1}(H)=\frac{21 n^{3} H^{3}}{2}+6\left(n^{3}-2 n^{2}\right) H \lambda^{2}+\left(-15 n^{3}+18 n^{2}\right) H^{2} \lambda .
$$

Differentiating (3.36) along $e_{1}$ and using (3.35), (3.30), (3.27), (3.25) and (3.36), we get

$$
\begin{aligned}
& {\left[\left(13 n^{3}+\frac{11 n^{2}}{2}\right) H^{3}+\left(4 n^{3}-14 n^{2}+2 n+20\right) H \lambda^{2}+\left(-15 n^{3}+18 n^{2}+24 n\right) H^{2} \lambda\right] \alpha} \\
& \quad+\left[-31 n^{2} H^{3}+\left(-16 n^{2}+36 n-8\right) H \lambda^{2}+\left(42 n^{2}-60 n\right) H^{2} \lambda\right] \beta \\
& \quad=e_{1}(H)\left[\frac{69 n^{2} H^{2}}{2}+\left(24 n-30 n^{2}\right) H \lambda+\left(6 n+4 n^{2}-28\right) \lambda^{2}\right] .
\end{aligned}
$$

Also, we have

$$
3 n e_{1}(H)=\alpha(n-2)(n H+2 \lambda)+\beta(-4 n H+2(n-2) \lambda)
$$

Combining (3.37) and (3.38), we obtain

$$
\begin{aligned}
& {\left[\left(9 n^{3}+171 n^{2}\right) H^{3}+\left(16 n^{3}+40 n^{2}-244 n-200\right) H \lambda^{2}+\left(-30 n^{3}-198 n^{2}-516 n\right) H^{2} \lambda\right.} \\
& \left.\quad-\left(16 n^{2}-8 n-160+\frac{224}{n}\right) \lambda^{3}\right] \alpha+\left[90 n^{2} H^{3}+\left(56 n^{2}-72 n-80\right) H \lambda^{2}\right. \\
& \left.\quad+\left(-126 n^{2}+108 n\right) H^{2} \lambda-\left(16 n^{2}-8 n-160+\frac{224}{n}\right) \lambda^{3}\right] \beta=0 .
\end{aligned}
$$


For simplicity, we denote by

$$
\begin{aligned}
p_{1}= & \left(9 n^{3}+171 n^{2}\right) H^{3}+\left(16 n^{3}+40 n^{2}-244 n-200\right) H \lambda^{2} \\
& +\left(-30 n^{3}-198 n^{2}-516 n\right) H^{2} \lambda-\left(16 n^{2}-8 n-160+\frac{224}{n}\right) \lambda^{3} \\
p_{2}= & 90 n^{2} H^{3}+\left(56 n^{2}-72 n-80\right) H \lambda^{2}+\left(-126 n^{2}+108 n\right) H^{2} \lambda \\
& -\left(16 n^{2}-8 n-160+\frac{224}{n}\right) \lambda^{3} .
\end{aligned}
$$

Therefore, (3.39) can be rewritten as

$$
\alpha p_{1}+\beta p_{2}=0
$$

On the other hand, combining (3.38) with (3.36) and using (3.35), we find

$$
\alpha^{2}(n-2)(10-2 n)(n H+2 \lambda)-4 \beta^{2}(-4 n H+2(n-2) \lambda)=R,
$$

where $\mathrm{R}$ is given by

$$
\begin{aligned}
R= & \frac{63 n^{3} H^{3}}{2}+\left(28 n^{3}-106 n^{2}+100 n\right) H \lambda^{2}+\left(102 n^{2}-51 n^{3}\right) H^{2} \lambda \\
& -\left(4 n^{3}-28 n^{2}+64 n-48\right) \lambda^{3} .
\end{aligned}
$$

Using (3.40) and (3.35), we get

$$
\alpha^{2}=-\frac{\lambda p_{2}}{p_{1}}\left(\frac{3 n H}{2}-(n-2) \lambda\right), \quad \beta^{2}=-\frac{\lambda p_{1}}{p_{2}}\left(\frac{3 n H}{2}-(n-2) \lambda\right)
$$

Eliminating $\alpha^{2}$ and $\beta^{2}$ from (3.41), we obtain

$$
\begin{aligned}
& \left(\frac{3 n H \lambda}{2}-(n-2) \lambda^{2}\right)\left[\left(14 n-2 n^{2}-20\right)(n H+2 \lambda) p_{2}^{2}-4 p_{1}^{2}(-4 n H+2(n-2) \lambda)\right] \\
& \quad=R p_{1} p_{2}
\end{aligned}
$$

which is a homogeneous equation of degree 9 in terms of $\lambda$ and $H$. Here, we point out that $\lambda \neq 0$. In fact, if $\lambda=0$ then (3.42) gives $H=0$, which is contradiction to our assumption. We put $Y=\frac{H}{\lambda}$, then (3.42) will reduce to an algebraic equation of degree 8 in $Y$

$$
\left(\frac{3 n Y}{2}-(n-2)\right)\left[\left(14 n-2 n^{2}-20\right)(n Y+2) q_{2}^{2}-4 q_{1}^{2}(-4 n Y+2(n-2)]=r q_{1} q_{2}\right.
$$


where

$$
\begin{aligned}
q_{1}= & \left(9 n^{3}+171 n^{2}\right) Y^{3}+\left(16 n^{3}+40 n^{2}-244 n-200\right) Y \\
& +\left(-30 n^{3}-198 n^{2}-516 n\right) Y^{2}-\left(16 n^{2}-8 n-160+\frac{224}{n}\right), \\
q_{2}= & 90 n^{2} Y^{3}+\left(56 n^{2}-72 n-80\right) Y+\left(-126 n^{2}+108 n\right) Y^{2} \\
& -\left(16 n^{2}-8 n-160+\frac{224}{n}\right), \\
r= & \frac{63 n^{3} Y^{3}}{2}+\left(28 n^{3}-106 n^{2}+100 n\right) Y+\left(102 n^{2}-51 n^{3}\right) Y^{2} \\
& -\left(4 n^{3}-28 n^{2}+64 n-48\right),
\end{aligned}
$$

and without having solve to (3.43) explicitly, even in the case of the existence of a real solution, $H$ will be proportional to $\lambda$ with a numerical factor $\mu$, where $\mu$ be the root of the equation (3.43). Hence, we can assume that $H=\mu \lambda$ and substituting it in (3.25), (3.27), (3.30), we obtain

$$
\begin{aligned}
& -2 e_{1} e_{1}(\lambda)+\frac{e_{1}^{2}(\lambda)}{\lambda}\left[\frac{4}{\mu n+2}+2\right]=\frac{n \mu(n \mu+2) \lambda^{3}}{2} \\
& e_{1} e_{1}(\lambda)+\frac{e_{1}^{2}(\lambda)}{\lambda}\left[\frac{3 n \mu-2 n+4}{-4 \mu n+2 n-4}-1\right]=\frac{n \mu(-4 n \mu+2 n-4) \lambda^{3}}{4}, \\
& -e_{1} e_{1}(\lambda)+\frac{e_{1}^{2}(\lambda)}{\lambda}\left[\frac{2(n-2)}{\mu n+2}+\frac{3 n \mu-2 n+4}{4 \mu n-2 n+4}\right] \\
& =-\lambda^{3}\left[\frac{n^{2} \mu^{2}}{4}+(n-2)+\left(\frac{3 n \mu}{2}-(n-2)\right)^{2}\right] .
\end{aligned}
$$

From (3.44)-(3.46), we find

$$
\begin{aligned}
& e_{1}^{2}(\lambda)=\frac{(4 n \mu-2 n+4)(n \mu+2) \lambda^{4}}{4}, \\
& e_{1}^{2}(\lambda)=\frac{\left(\frac{7 \mu^{2} n^{2}}{2}-\frac{\mu n^{2}}{2}+n \mu(7-3 n)+(n-2)+(n-2)^{2}\right)(\mu n+2) \lambda^{4}}{\mu n-2 n+6} .
\end{aligned}
$$

Using (3.47) and (3.48), we get $\left(5 n^{2} \mu^{2}-2 \mu n^{2}-2 n-8\right)(n \mu+2)=0$. Since $\lambda \neq \frac{-n H}{2}$, gives $n \mu+2 \neq 0$. Therefore, we have

$$
5 n^{2} \mu^{2}-2 \mu n^{2}-2 n-8=0 .
$$

On the other hand, differentiating (3.47) along $e_{1}$, we obtain

$$
2 e_{1} e_{1}(\lambda)=(4 n \mu-2 n+4)(n \mu+2) \lambda^{3},
$$

From (3.44), (3.47), and (3.39), we find

$$
5 n \mu-2 n+6=0,
$$

which gives $\mu=\frac{2 n-6}{5 n}$. However, this contradicts the equation (3.49). 
Now, we have main result as follows:

THEOREM 3.2. There exist no proper bi-harmonic hypersurfaces with three distinct principal curvatures in the Euclidean space of arbitrary dimension.

AcKnowledgements. The author is grateful to Prof. A. Sharfuddin for his valuable suggestions and incessant motivations. Also, the author is thankful to the reviewer(s) for various remarks to improve the original version of the article.

\section{REFERENCES}

1. B. Y. Chen, Total mean curvature and submanifolds of finite type (World Scientific, Singapore, 1984).

2. B. Y. Chen, Some open problems and conjectures on submanifolds of finite type, Soochow J. Math. 17 (1991), 169-188.

3. B. Y. Chen, Submanifolds of finite type and applications, in Proc. Geometry and Topology Research Center, Taegu, vol. 3 (1993), 1-48.

4. B. Y. Chen, A report on submanifolds of finite type, Soochow J. Math. 22 (1996), $117-337$.

5. B. Y. Chen and M. I. Munteanu, Biharmonic ideal hypersurfaces in Euclidean spaces, Differ. Geom. Appl. 31 (2013), 1-16.

6. F. Defever, Hypersurfaces of $E^{4}$ with harmonic mean curvature vector, Math. Nachr. 196 (1998), 61-69.

7. I. Dimitric, Quadratic representation and submanifolds of finite type, Doctoral Thesis (Michigan State University, 1989).

8. I. Dimitric, Submanifolds of $E^{m}$ with harmonic mean curvature vector, Bull. Inst. Math. Acad. Sin. 20 (1992), 53-65.

9. Th. Hasanis and Th. Vlachos, Hypersurfaces in $E^{4}$ with harmonic mean curvature vector field, Math. Nachr. 172 (1995), 145-169.

10. $\mathrm{Yu}$. Fu, Biharmonic hypersurfaces with three distinct principal curvatures in the Euclidean 5-space, J. Geom. Phys. 75 (2014), 113-119. 